\title{
A Prostate Brachytherapy Training Rehearsal System - Simulation of Deformable Needle Insertion
}

\author{
Asako Kimura (Nishitai) ${ }^{1,2}$, Jon Camp ${ }^{2}$, Richard Robb ${ }^{2}$, and Brian Davis ${ }^{3}$ \\ ${ }^{1}$ Graduate School of Engineering Science, Osaka University, \\ 1-3 Machikaneyama-cho, Toyonaka 560-8531, Japan \\ asa@sys.es.osaka-u.ac.jp \\ ${ }^{2}$ Biomedical Imaging Resource, Mayo Foundation, \\ Rochester, MN 55902, U.S.A \\ \{nishitai.asako,jjc,rar\}@mayo.edu \\ http: //www. mayo.edu/bir/ \\ ${ }^{3}$ Radiation Oncology, Mayo Foundation, \\ Rochester, MN 55902, U.S.A \\ davis.brian@mayo.edu
}

\begin{abstract}
Prostate cancer is the most common cancer diagnosed in men in the United States. Prostate brachytherapy - an advanced radiotherapy cancer treatment in which radioactive seeds are placed directly in and around the prostate tissues - is complicated by tissue forces that tend to deflect the treatment needles from their intended positions. Medical simulation has proven useful as a technique to enhance surgeons' training and procedure rehearsal. Therefore we are developing a brachytherapy simulation system that includes visual display and haptic feedback, with which a user can see and feel the procedure of the therapy. In this paper, we describe a deformable needle insertion simulation that is a part of our brachytherapy simulation system.
\end{abstract}

\section{Introduction}

Since high resolution tomographic image scanners, high performance imaging hardware and comprehensive medical imaging software have become available, interactive $3 \mathrm{D}$ visualization of the body, including all organs and their functions is used for accurate anatomy and function mapping, enhanced diagnosis, accurate treatment planning and rehearsal, and education/training [1][2]. Medical simulation is useful to enhance surgeon training and procedure rehearsal. It may also reduce costs and allay ethical concerns. For example, spinal nerve blocks [3], microsurgery [4], endoscopic surgery [5] and catheter insertion [6], can be faithfully simulated using highresolution graphics, calculated deformation of tissue and organs and haptic feedback.

For a medical simulation system to be useful, realism is one of the most essential factors to consider and provide. However, easy understanding of procedure and intuitive interface, and practice and repeat difficult and critical parts of procedures are also very important. In addition, by measuring and recording the hand movement and associated forces during a simulation, it is possible to make a database of each user's 
technique and habits. This database could help trainees to check whether their hand movement and force is the same as experts. Our objective is to make the simulation system very realistic. From this point of view, we are constructing a brachytherapy simulation system that faithfully incorporates the common tasks used in brachytherapy. The needle insertion procedure in prostate brachytherapy is not very complex, but requires training and skill. Thus this feature is the first step to realize our eventual objective of a complete brachytherapy procedure simulation. In this paper, we describe only this part of the system, namely our deformable needle insertion simulation.

\section{Brachytherapy}

In the United States, there were 189,000 new cases of prostate cancer estimated during 2002, and 30,200 deaths in 2002 making it the second leading cause of cancer death in men [7]. Since there is an excellent blood test, prostate-specific antigen (PSA), prostate cancer can be diagnosed at a very early stage. Brachytherapy is an advanced cancer treatment using radioactive seeds or sources placed in the tumor itself, giving a high radiation dose to the tumor while reducing the dose to surrounding healthy tissues in the body. Prostate cancer is well suited to it, since the prostate gland is nestled under the bladder and in front of the rectum. This anatomic arrangement makes external beam radiotherapy difficult, and it is imperative that the radiation be focused on the prostate to avoid serious side effects [8].

\subsection{Sequence of Brachytherapy - Operation Implant}

A prostate brachytherapy includes a three-step process: (1) pretreatment planning, (2) operative implant, and (3) post implant quality evaluation [9]. Since our training rehearsal system supports operative implant procedure, we will explain this procedure in detail.

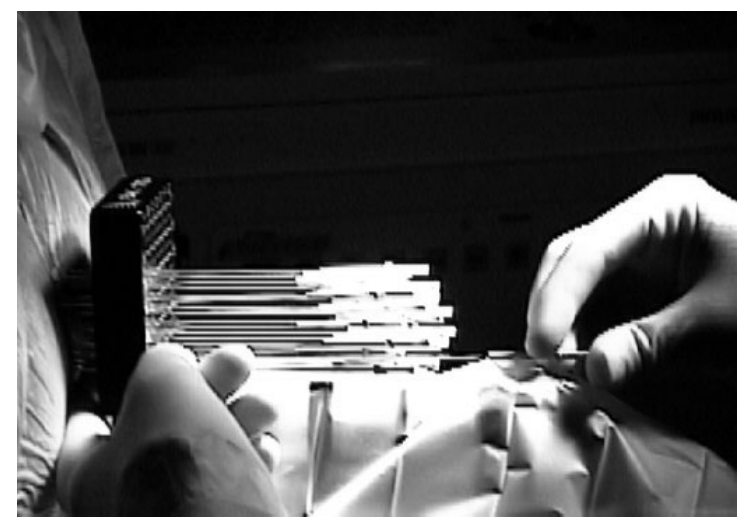

Fig. 1. Brachytherapy Needle Insertion 
1) Setting: A fluoroscope, a rectal ultrasound probe in a patient body. The rectal ultrasound is movable so that doctors can check all inserted radiation seed positions, three inches square template is attached to the ultrasound probe. The template has holes every $0.5 \mathrm{~cm}$ through which doctors place needles.

2) Calibration: Two non-radioactive seeds are injected as reference markers for calibration between pre-planned prostate position and current prostate position.

3) Needle insertion (Fig.1): Insert hollow needles with a removable metal insert. They are placed through the template, through the skin, and into the prostate gland. The doctors use the ultrasound monitor to check whether the needles are being placed into the correct position or not.

4) Seed injection: Inject seeds throw hollow needles. As during needle insertion, the doctors use the fluoroscopy monitor to check whether the seeds are being injected to the correct position or not.

\subsection{Needle Deflection}

Since it is imperative in prostate brachytherapy that the radiation is focused not on the other organs but only on the prostate to avoid serious side effects, precise needle positioning is of primary importance.

The problem of needle insertion is needle deflection. It is not easy for surgeons to precisely reach the planned target inside soft tissue with a needle because a thin needle can be deflected in the tissue and the needle also can deform the tissue itself, even if surgeons attempt to lead the needle in a straight direction [10]. Moreover, since the needle path encounters many different tissues of varying resilience, the net deflection is difficult to predict. Therefore a simulation system of a deformable needle insertion is helpful for surgeons and residents for training and rehearsal.

\section{Materials and Methods}

\subsection{System Overview}

Our Brachytherapy training rehearsal system consists of a display server and a haptic server (Fig.2). The display server is a SGI workstation (SGI OCTANE) operating under the IRIX6.5. Users can see 3D images of the prostate and other organs, a fluoroscopy image and an ultrasound image on the SGI display (Fig.3). All 3D models of organs are constructed using the medical imaging software Analyze developed in our laboratory, and visualized using OpenGL.

The haptic server is a PC (DELL Precision620) operating under the Windows 2000. We use a PHANToM (PHANTOM Premium 1.5) as a haptic device and GHOST SDK 3.1 as programming software to simulate haptic feedback for needle insertion. In our system, we put a real needle used in a brachytherapy procedure on the end of the PHANToM's stick. 


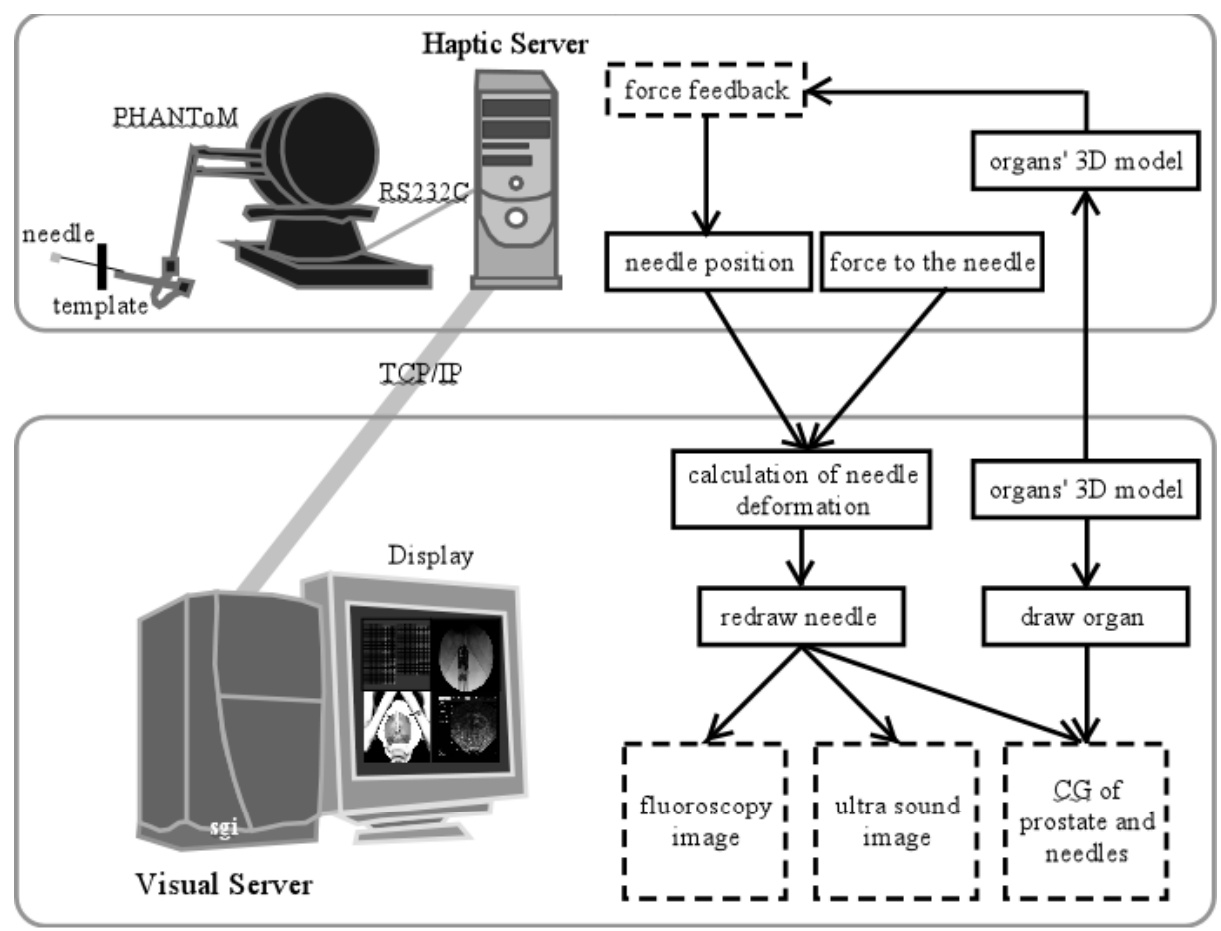

Fig. 2. System architecture

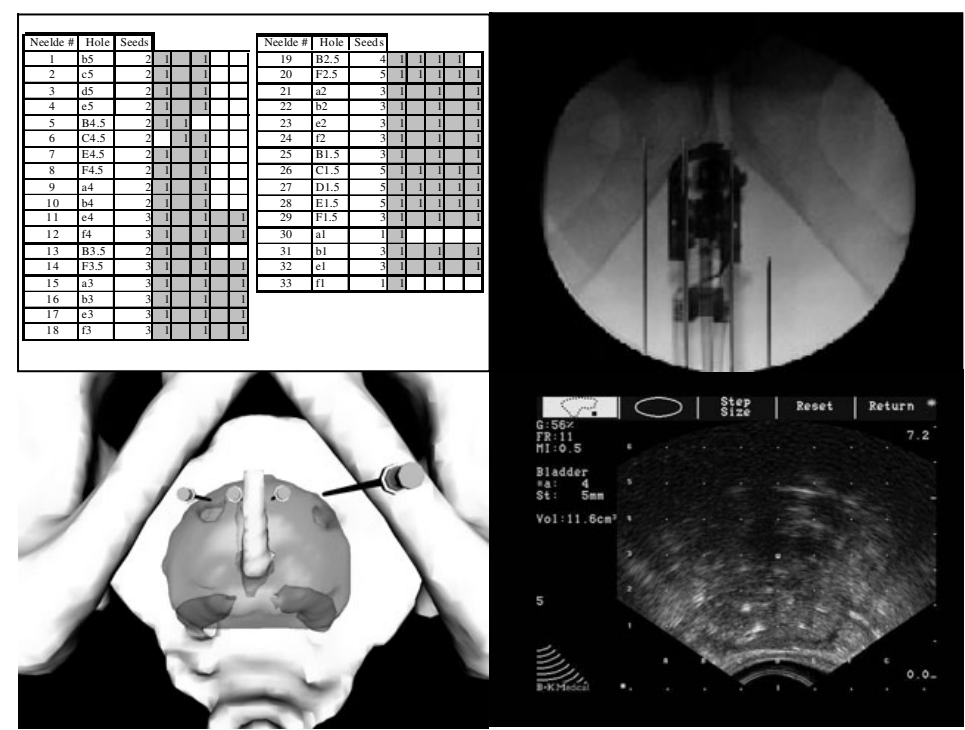

Fig. 3. Visual Display: upper left is planned seed positions, upper right is a fluoroscope image, lower left is a CG of prostate and needles, and lower right is ultrasound image 
To synchronize visual needle insertion and haptic feedback, the haptic server sends the latest 3D position of the needle to visual server over TCP/IP. The haptic server also sends user's force to the needle to visual server to simulate needle deformation. This network communication is established with CORBA (AT \& T omniORB3.04), the software for distributed computing.

\subsection{Simulation of Needle Deflection}

\section{Visual Feedback}

The prostate lies behind layers of fat, muscle, and soft tissue (Fig.4.). When the needles pass each layer, they encounter varying forces that may gradually deflect them. In addition, the prostate and surrounding tissues are soft, so that the prostate itself is movable. This prostate movement also causes needle deflection. It is very hard to model this needle deflection exactly, since size of prostate, density and thickness of each layer and prostate motility differs widely between patients. Surgeons cannot always estimate how the needle will deform before the insertion, but they check how the needle has deformed, and then remove it halfway and reinsert it. Therefore, our focus is on modeling the appropriate magnitude and direction of deflection forces, rather than predicting the exact deflection forces that a particular insertion path will encounter.

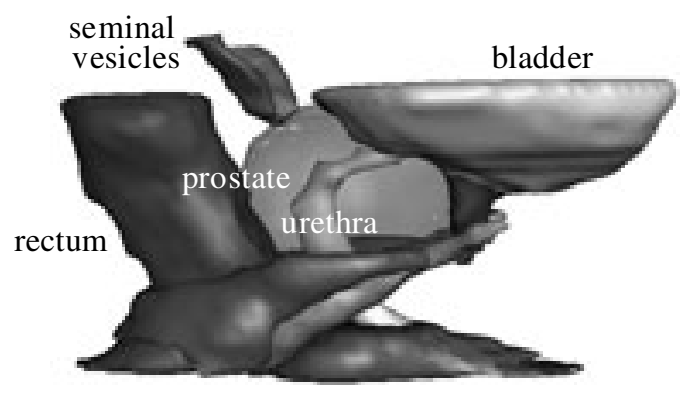

(a) Anatomy of Prostate

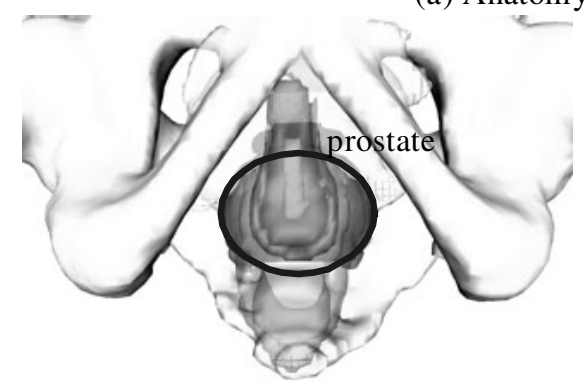

(b) Supine

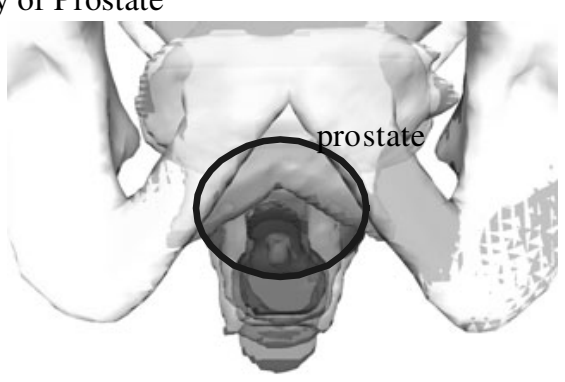

(c) Prone

Fig. 4. Anatomy of prostate: Many layers of different structures and density cause needle deflection 
In our initial needle insertion simulation, when the needle passes a new layer, tissues around the needle apply a fixed force. This force is randomly to each layer, and these random series of forces may or may not produce significant needle deflection. On reinsertion, the needle deformation is recalculated based on the previous needle deflection, combined with the force direction and magnitude with which the user pushes the needle to correct the needle position.

In our system, the needle deflection is visible in the ultrasound image and the fluoroscopy image. In a real needle insertion procedure, surgeons don't check needle deflection using the fluoroscopy image to reduce radiation from the fluoroscopy. However, since the fluoroscopy image can show the whole needle deflection image, the user can find needle deflection easier by using both of them. Therefore, in our system, the user can use both images at first, and after they are more experienced, they can turn off the fluoroscopy image.

Fig.5 shows results of needle deflection simulation in the ultrasound image and fluoroscopy image.
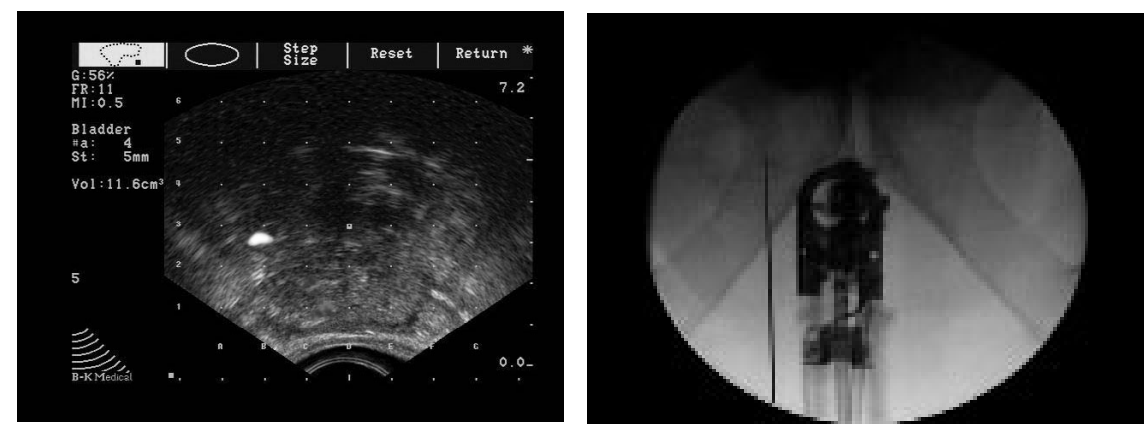

Fig. 5 Visual simulation result of deflected needle in the ultrasound image (left) and a fluoroscopy image (right): A white spot in the ultrasound image is the tip of the needle

\section{Haptic Feedback}

Fig.6 shows the haptic feedback in our system. Two kinds of haptic feedback are presented; one is to fingers that push needle into prostate, and another is to fingers of the other hand that corrects needle deflection. When the needle passes each surface between organs, a haptic feedback of puncture is provided to the fingers pushing the needle. During needle reinsertion, half of the needle is already fixed with skin and muscles. Therefore to correct needle deflection, surgeons push on the middle of the needle behind the template with fingers of the other hand. This time, force feedback is presented to the fingers in the opposite direction that the user pushes.

\section{Evaluation}

We are planning to evaluate our training rehearsal system from two aspects; the reality and the usefulness for training. 


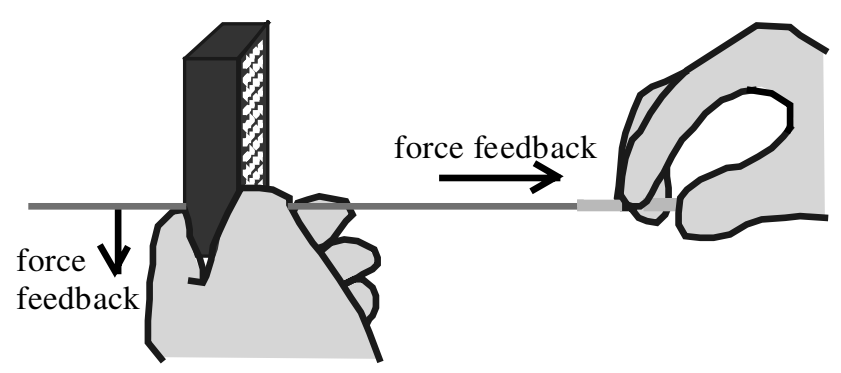

Fig. 6. Two kinds of haptic feedback are presented; one is to fingers that push the needle into prostate, and another is to fingers that correct needle deflection

Firstly, we will have experienced urologists assessed the realism of force magnitudes and needle deformation width and visualization. We will then improve haptic feedback and visual simulation of our initial system.

Secondly, we will evaluate improvement of residents' performance by using our training rehearsal system. Using our system, we will have experienced urologists judge whether needle position is good enough or not. Since our system can measure and save final deflection of the needle, we will gather all data of distance from planned needle positions to inserted needles position, and average them. Based on this result, all new needle deflection can be detected whether their positions are near enough from the planned position. By using this detection, both residents' needle positioning quality and improvement of residents' performance after using our system can be assessed. We will assess improvement of three residents' performance by comparing results between first trial and third trial of our system. We will also discuss usability of our system from the results and the comments from the residents.

\section{Conclusions and Future Plans}

By using our deformable needle insertion training and rehearsal system, surgeons and residents can train and rehearse needle insertion and correction of deflected needle position by three visual feedbacks, CG of prostate and needles, the ultrasound image and fluoroscopy image, and haptic feedback. We are planning to evaluate realism and usefulness of our training rehearsal system by experienced urologists and residents. Our next step is to complete these two evaluations and to add a seed injection training procedures.

\section{Acknowledgements}

We thank Bruce Cameron (Biomedical Imaging Resource, Mayo Foundation, Rochester) for technical advice about network computing and biomedical image visualization. 


\section{References}

1. R.A. Robb (ed.): THREE-DIMENTIONAL BIOMEDICAL IMAGING. A John Wiley \& Sons, Inc. (1998)

2. R.A. Robb (ed.): BIOMEDICSL IMAGING, VISUALIZATION, and ANALYSIS. A John Wiley \& Sons, Inc. (2000)

3. D.J. Blezek, R.A. Robb, J.J. Camp, L.A. Nauss, D.P. Martin: Simulation of spinal nerve blocks for training anesthesiology residents. Proceedings of Surgical-Assist Systems, BiOS 98 (1998) 45-51

4. J. Brown, K. Montgomery, J. Latombe, and M. Stephanides: A Microsurgery Simulation System. MICCAI 2001, LNCS2208 (2001) 137-144

5. C. Baur, D. Guzzoni, and O. Georg: A Virtual Reality and Force Feedback Based Endoscopic Surgery Simulator. MMVR1998 (1998) 110-116

6. Zorcolo, E. Gobbetti, G. Zanetti, and M. Tuveri: Catheter insertion simulation with coregistered direct volume rendering and haptic feedback. MMVR2000 (2000) 96-98

7. Cancer Facts and Figures. American Cancer Society (2002) http://www.cancer.org/downloads/STT/CFF2002.pdf

8. http://www.brachytherapy.com/

9. H. RAGDE: Brachytherapy (Seed Implantation) for Clinically Localized Prostate Cancer. Journal of Surgical Oncology, Vol64 (1997) 79-81

10. H. Kataoka, T. Washio, M. Audette, and K. Mizuhara: A Model for Relations between Needle Deflection, Force, and Thickness on Needle Penetration. MICCAI 2001, LNCS2208 (2001) 966-974 